\title{
Phonon anharmonicities in supported graphene
}

\author{
Egor A. Kolesov a , Mikhail S. Tivanov ${ }^{\text {a, }}{ }^{\text {, }}$, Olga V. Korolik ${ }^{\text {a }}$, Olesya O. Kapitanova ${ }^{\text {b }}$, \\ Hak Dong Cho ${ }^{c}$, Tae Won Kang ${ }^{c}$, Gennady N. Panin ${ }^{\text {c, d }}$ \\ a Belarusian State University, 4 Nezavisimosti Av., 220030 Minsk, Belarus \\ b Department of Chemistry, Moscow State University, Leninskie Gory, 1, b.3, 119991, Moscow, Russia \\ c Department of Physics, Quantum-Functional Semiconductor Research Center, Nano Information Technology Academy, Dongguk University, 3-26 Pildong, \\ Junggu, 100-715, Seoul, South Korea \\ ${ }^{\mathrm{d}}$ Institute for Microelectronics Technology \& High Purity Materials, RAS, 142432 Chernogolovka, Moscow District, Russia
}

\section{A R T I C L E I N F O}

\section{Article history:}

Received 1 June 2018

Received in revised form

28 August 2018

Accepted 3 September 2018

Available online 7 September 2018

\begin{abstract}
A B S T R A C T
The paper presents temperature-dependent Raman studies of anharmonic phonon properties of graphene as-grown on copper, transferred to copper, $\mathrm{SiO}_{2} / \mathrm{Si}$, and $\mathrm{Al}_{2} \mathrm{O}_{3}$, as well as nitrogen-doped graphene on $\mathrm{SiO}_{2} / \mathrm{Si}$. Different $\mathrm{G}$ and $2 \mathrm{D}$ peak position and linewidth temperature dependencies were obtained in the temperature range of $20-294 \mathrm{~K}$, upon which anharmonic constants for 3- and 4-phonon processes were determined. Values of anharmonic constants obtained from $\mathrm{G}$ peak shift for undoped graphene on dielectric substrates were quantitatively close to both experimental results for unsupported graphene and theoretical predictions reported in the literature, while the values for graphene as-grown on copper were almost two orders of magnitude greater. The results were analyzed in terms of substrate effect on phonon properties of graphene. The present study is useful for taking into account anharmonic phonon effects in graphene when designing graphene-based nanoelectronic devices.
\end{abstract}

(C) 2018 Elsevier Ltd. All rights reserved.

\section{Introduction}

Due to the unique physical properties of graphene, it is a promising material for a variety of applications [1]. In particular, it demonstrates high values of carrier mobility and thermal conductivity [1-4]. Electrical and thermal transport in graphene are known to be strongly affected by anharmonic phonon-related effects such as electron-phonon coupling [5-7] or high-order phonon-phonon interactions [8-11], including coupling of optical and acoustic phonon modes. Consequently, these processes are directly related to such common problems of nanoelectronics as increasing electrical conductivity or removing heat from the device functional elements, as well as applied tasks such as determining graphene thermal conductivity [8,12], which underlines the importance of investigating the anharmonic phonon properties of graphene.

At the same time, various nanoelectronic applications require graphene layers supported by the substrates, which in turn affect physical properties of the two-dimensional material. Graphene

\footnotetext{
* Corresponding author.

E-mail address: tivanov@bsu.by (M.S. Tivanov).
}

phonon dispersion is very sensitive to different interatomic forces such as graphene-substrate interaction [13]. As it was shown in Ref. [14] for graphene on such a common substrate as $\mathrm{SiO}_{2} / \mathrm{Si}$, coupling of acoustic phonons to the substrate Rayleigh waves may linearize the dispersion, increasing hybridized mode group velocity. Phonons can leak through the interface [15] or scatter on it [16]. As graphene gets adsorbed on the substrate surface, modification of graphene phonon dispersion can be significant; it will expectedly lead to stronger anharmonic effects in atom oscillations, making an experimental study of supported graphene phonon properties relevant.

Raman spectroscopy is a powerful tool for graphene studies, being a versatile non-destructive method to obtain information about graphene phonon properties [17]. The purpose of the present study is to experimentally determine and analyze anharmonic phonon temperature shifts and broadening for supported graphene by Raman spectroscopy in order to establish the substrate effect on the anharmonic phonon properties of graphene.

\section{Experimental}

Graphene was synthesized on copper foil at $1020^{\circ} \mathrm{C}$ by chemical vapor deposition (CVD) with $\mathrm{CH}_{4}$ flow of $40 \mathrm{sccm}$ and hydrogen 
flow of $10 \mathrm{sccm}$. Copper foil (Alfa Aesar, 99.999\%, $10 \times 30 \mathrm{~cm}^{2}$ $25 \mu \mathrm{m}$ thick) was pre-annealed at $1060^{\circ} \mathrm{C}$ under hydrogen flow of $300 \mathrm{sccm}$ and argon flow of $2000 \mathrm{sccm}$ at a pressure of $<10^{-4}$ Torr for $1-2 \mathrm{~h}$ inside the chamber. After graphene growth, $\mathrm{Cu}$ foil was cooled down to room temperature. The cooling rate was described in detail elsewhere [18].

Graphene was transferred to various substrates using "PMMAmediated" method [19]. PMMA (molecular weight $=996000$, dissolved in anisole) was spin-coated (3000 rpm, $1 \mathrm{~min}$ ) on graphene supported by copper foil. An $0.1 \mathrm{M}\left(\mathrm{NH}_{4}\right)_{2} \mathrm{~S}_{2} \mathrm{O}_{8}$ aqueous solution was used for copper etching, and water/isopropyl alcohol mixture was used in order to remove the etching products from graphene [20]. PMMA was removed by submerging the sample in glacial acetic acid (extra pure) [21] for $4 \mathrm{~h}$.

Graphene, doped with nitrogen, was prepared using N-plasma treatment described elsewhere [22].

Raman spectra were obtained using Nanofinder HE (LOTIS TII) confocal Raman spectrometer with a spectral resolution better than $3 \mathrm{~cm}^{-1}\left(\sim 2.3 \mathrm{~cm}^{-1}\right.$ for $\mathrm{G}$ peak and $\sim 1.0 \mathrm{~cm}^{-1}$ for 2D peak). For excitation of Raman radiation, a continuous solid-state laser with a wavelength of $473 \mathrm{~nm}$ was used. Raman measurements were carried out using laser radiation power at the optical system output of $2.4 \mathrm{~mW}$, laser spot diameter was of $0.6 \mu \mathrm{m}$ during roomtemperature measurements.

Using low-temperature range for the measurements traditionally allows to exclude undesirable temperature effects (for example, on graphene-substrate interaction) in order to focus more on fundamental properties of the system. During the lowtemperature measurements, the sample was studied in a vacuum (less than $5 \times 10^{-4} \mathrm{~Pa}$ ) temperature-controlled cell. The laser spot diameter was of $\sim 1.5 \mu \mathrm{m}$. The measurements were performed in the temperature range from 20 to $294 \mathrm{~K}$ with a setting accuracy of $\pm 0.05 \mathrm{~K}$.

\section{Results and discussion}

Room-temperature Raman spectra of the experimental graphene are presented in Fig. 1, where $G$ and 2D features are observed, typical for this material [17]. The absence of a distinguishable D peak in the spectra of undoped graphene indicates low defect density [17]; for nitrogen-doped graphene, G peak position is upshifted by $\sim 3 \mathrm{~cm}^{-1}$, with that of $2 \mathrm{D}$ being normal, indicating electron doping together with $2 \mathrm{D}$ and $\mathrm{G}$ peak intensity ratio of 1.4-1.6 [23]. Another disorder-induced band $\mathrm{D}^{\prime}$ is also present in this case. $G$ and $2 D$ peak positions for as-grown graphene on copper are upshifted by $20-26 \mathrm{~cm}^{-1}$, which is indicative for the presence of substrate-induced strain [24] generated during the cooling stage of the synthesis process, when copper substrate contracts under the as-grown graphene.

Fig. 2 demonstrates $G$ and 2D peak position temperature shifts for the experimental graphene. As the temperature decreases from 294 to $20 \mathrm{~K}$, a different total peak upshift for graphene on different substrates is observed: from $3-7 \mathrm{~cm}^{-1}$ on $\mathrm{Al}_{2} \mathrm{O}_{3}$ to $18-42 \mathrm{~cm}^{-1}$ on copper (as-grown). The obtained shifts are generally stronger than those reported for free-standing graphene [25-27]. Presence of several slightly upshifted points in Fig. 2 at temperatures close to $300 \mathrm{~K}$ (for example, graphene on copper) can be attributed to the initial atmospheric doping of graphene [28] (though each sample was situated in a vacuum cell with the pump operating for at least $3 \mathrm{~h}$ before the experiment, it is possible that a small number of adsorbates was still present on the sample surface until further removal due to the energy transfer from the laser radiation).

Usually, the linear approximations of the presented dependencies are encountered in the literature. Straight line fit results are given in Appendix A for comparison. Solid curves in Fig. 2 show the results of a model calculation presented below.

The phonon mode energy temperature shift is one of the major manifestations of anharmonic phonon effects. Experimental $G$ peak

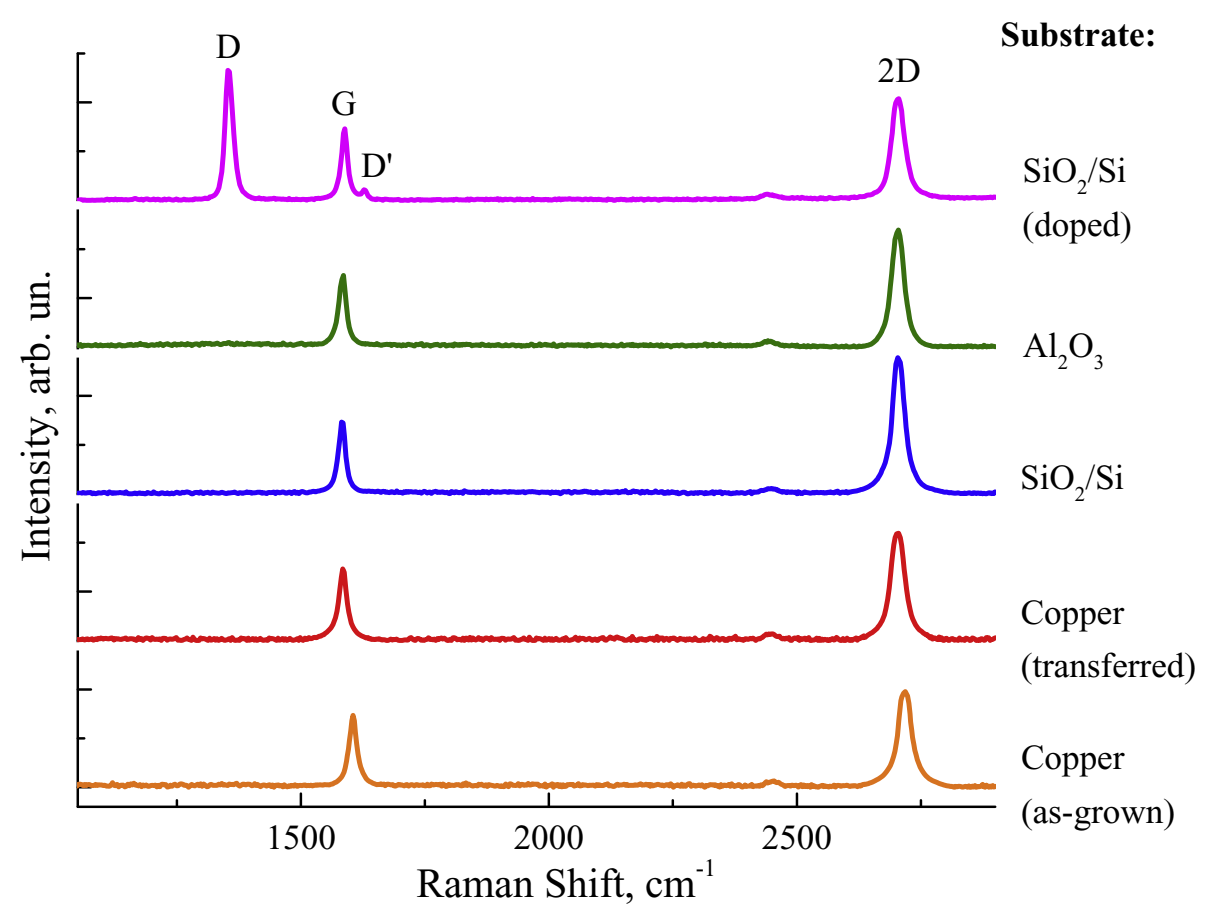

Fig. 1. Typical Raman spectra of graphene on various substrates, as well as nitrogen-doped graphene. (A colour version of this figure can be viewed online.) 

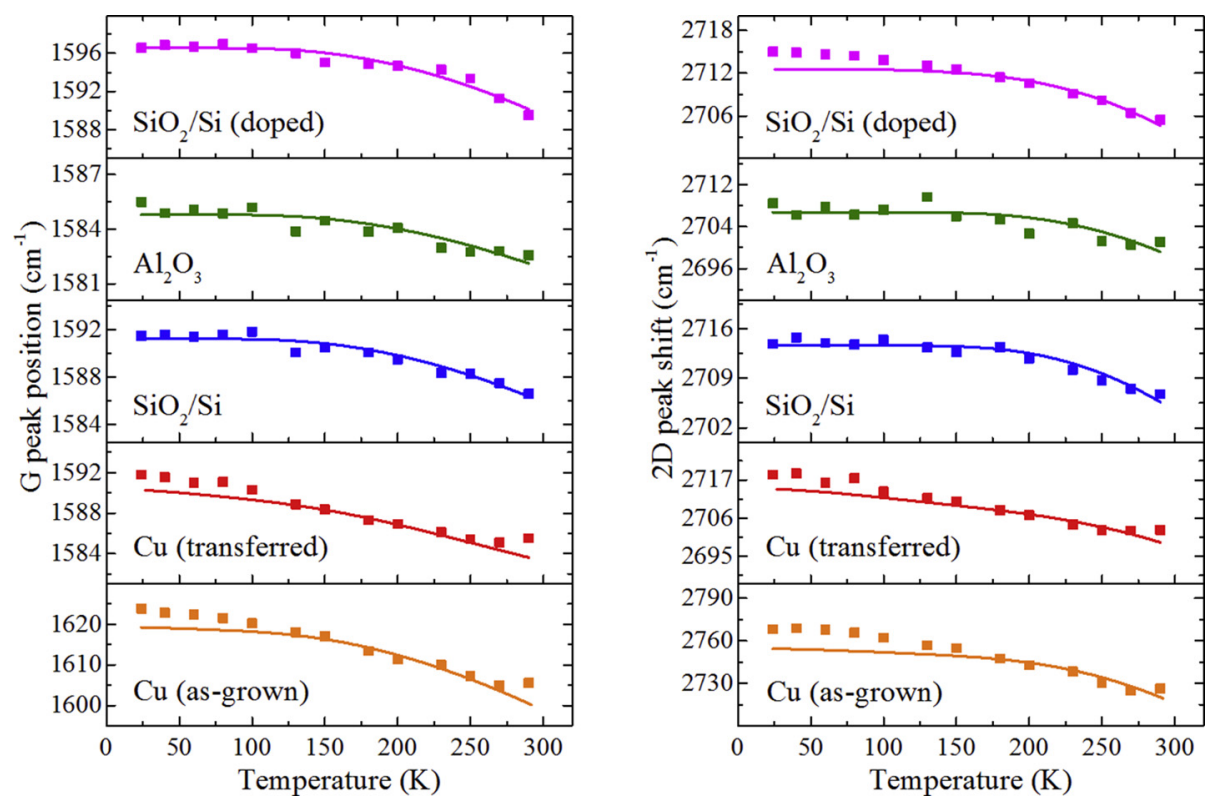

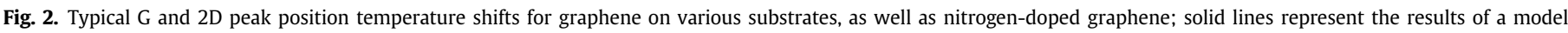
calculation. (A colour version of this figure can be viewed online.)

position variations $\Delta \omega_{\exp }^{G}$ with respect to a normal position are defined by anharmonic phonon-phonon processes $\Delta \omega_{p h-p h}^{G}[29,30]$; graphene thermal expansion $\Delta \omega_{V}^{G}$; graphene deformation caused by the thermal expansion coefficient (TEC) difference for graphene and substrate $\Delta \omega_{\text {str }}^{G}$ [25,31]; electron-phonon coupling (EPC) $\Delta \omega_{e l-p h}^{G}[32]$ and atmosphere-induced doping $\Delta \omega_{\text {doping }}^{G}$ [28]. Thus, the overall shift of a phonon mode energy can be estimated by the following expression [25,31]:

$\Delta \omega_{\text {exp }}^{G}=\Delta \omega_{p h-p h}^{G}+\Delta \omega_{V}^{G}+\Delta \omega_{\text {str }}^{G}+\Delta \omega_{\text {el-ph }}^{G}+\Delta \omega_{\text {doping }}^{G}$,

Since 2D peak is a Raman overtone of $\mathrm{D}$ peak and corresponds to the same "breathing" vibration [17], thermal expansion contribution $\Delta \omega_{V}^{2 D}$ in its temperature shift can be considered trivial [25]. Thus, the shift includes phonon-phonon, substrate-induced strain, electron-phonon, and doping contributions:

$\Delta \omega_{\exp }^{2 D}=\Delta \omega_{p h-p h}^{2 D}+\Delta \omega_{\text {str }}^{2 D}+\Delta \omega_{e l-p h}^{2 D}+\Delta \omega_{\text {doping }}^{2 D}$

All corresponding terms of equation (2) for 2D peak can be defined in a similar way to those for G peak [25,32]. The $\Delta \omega_{p h-p h}^{G, 2 D}$ term can be calculated using a Klemens model considering threeand four-phonon interactions, which was originally developed for the phonon linewidth but can also be applied to describe the lineshift through the following equation [25]:

$$
\begin{aligned}
\Delta \omega_{p h-p h}^{G, 2 D}= & A_{3-p h}^{G, 2 D}\left[1+\frac{2}{\exp \left(\frac{\hbar \omega_{0}^{G, 2 D}}{2 k T}\right)-1}\right] \\
& +B_{4-p h}^{G, 2 D}\left[1+\frac{3}{\exp \left(\frac{\hbar \omega_{0}^{G, 2 D}}{3 k T}\right)-1}+\frac{3}{\left(\exp \left(\frac{\hbar \omega_{0}^{G, 2 D}}{3 k T}\right)-1\right)^{2}}\right],
\end{aligned}
$$

where the coefficients $A_{3-p h}^{G, 2 D}$ and $B_{4-p h}^{G, 2 D}$ are constants, representing the contributions of three-phonon and four-phonon processes to the frequency shift, respectively. Extracting the values of these constants from the measurements for supported graphene provides an important information on how strongly the substrate material affects graphene phonon properties. It should be noted that the modification of phonon dispersion by graphene-substrate interaction does not fundamentally affect the nature of the anharmonic process, which is described by the exponential parts of equation (3). At the same time, the effect of the substrate on graphene force constants and atomic displacements will be expressed in the altered values of anharmonic constants $A_{3-p h}^{G, 2 D}$ and $B_{4-p h}^{G, 2 D}$, obtained as output approximation parameters.

The term arising in (1) due to graphene lattice thermal expansion/contraction is given by Ref. [31]:

$$
\begin{aligned}
\Delta \omega_{V}^{G}=\omega_{V}^{G}\left(T_{1}\right)-\omega_{V}^{G}\left(T_{2}\right)= & \omega_{0}^{G}\left[\exp \left(-3 \gamma_{G} \int_{0}^{T_{1}} \alpha_{\text {graph }}\left(T^{\prime}\right) d T^{\prime}\right)\right. \\
& \left.-\exp \left(-3 \gamma_{G} \int_{0}^{T_{2}} \alpha_{g r a p h}\left(T^{\prime}\right) d T^{\prime}\right)\right]
\end{aligned}
$$

where $\gamma_{G}$ is a Grüneisen parameter for $E_{2 g}$ optical phonon at the Brillouin zone center (value of 1.8 from Ref. [24] was used in this paper), $\alpha_{\text {graph }}\left(T^{\prime}\right)$ is graphene TEC as a function of temperature $T^{\prime}$. As it was shown in Ref. [33], $\alpha_{\text {graph }}\left(T^{\prime}\right)$ strongly depends on graphenesubstrate interaction energy both quantitatively and qualitatively. In order to choose correct graphene TEC temperature dependence [33] for each substrate, we used total interaction energy values of $0.72,0.47,0.45 \mathrm{~J} / \mathrm{m}^{2}$ for graphene on copper, $\mathrm{Al}_{2} \mathrm{O}_{3}$ and $\mathrm{SiO}_{2} / \mathrm{Si}$, respectively [34,35]. Despite the stronger graphene-copper interaction can be expected for an as-grown material than for a transferred one (for example, due to possible presence of the additional 
chemical bonding at the interface [36]), we took similar interaction energy values for both cases based on similarity of results reported in the literature for both self-equilibrium theoretical calculations taking into account van der Waals and strain effects [34] and experimental adhesion energy measurements for as-grown graphene on copper [37]. Utilizing substrate-dependent graphene TEC dependencies from Ref. [33] leads to generally small influence of thermal expansion contributions, weakly compensating the whole $G$ peak position dependency in agreement with [25] - from below $\sim 2 \mathrm{~cm}^{-1}$ in case of $\mathrm{SiO}_{2} / \mathrm{Si}$ substrate to maximal $\sim 1 \mathrm{~cm}^{-1}$ for copper substrate.

Mechanical strain influence in (1) and (2) caused by the difference of graphene and substrate TECs can be written as [38]:

$\Delta \omega_{\text {str }}^{G, 2 D}=\beta_{G, 2 D} \int_{T_{1}}^{T_{2}}\left(\alpha_{\text {sub }}\left(T^{\prime}\right)-\alpha_{\text {graph }}\left(T^{\prime}\right)\right) d T^{\prime}$

where $\beta_{G, 2 D}=\partial \omega_{G, 2 D} / \partial \varepsilon$ is a biaxial strain rate (the values of $\beta_{G}=-$ $58 \frac{\mathrm{cm}^{-1}}{\%}$ and $\beta_{2 D}=-144 \frac{\mathrm{cm}^{-1}}{\%}$ from Ref. [24] were used in the present study), $\varepsilon$ is a relative deformation (\%), $\alpha_{s u b}\left(T^{\prime}\right)$ is the thermal expansion coefficient as a function of temperature $T^{\prime}$ for the substrate. The calculations give the maximum values of $\Delta \omega_{s t r}^{G}$ at $210-220 \mathrm{~K}$ ranging from $\sim 3$ to $\sim 2 \mathrm{~cm}^{-1}$ for $\mathrm{Cu}$ and $\mathrm{SiO}_{2} / \mathrm{Si}$ substrates, respectively. For $\Delta \omega_{s t r}^{2 D}$, maximum values change from $\sim 5$ to $\sim 2 \mathrm{~cm}^{-1}$.

EPC contribution to the temperature shift $\Delta \omega_{e l-p h}^{G, 2 D}$ was calculated in Ref. [32] using an extensive theoretical model. The results given in Ref. [32] show its value changing by about $3 \mathrm{~cm}^{-1}$ as the temperature rises from a value close to absolute zero to about $300 \mathrm{~K}$ $\left(\sim 4 \mathrm{~cm}^{-1}\right.$ for $2 \mathrm{D}$ peak).

Low-pressure environment naturally provides a minimal temperature variation of atmosphere-induced doping contribution $\Delta \omega_{\text {doping. }}^{G, 2 D}$. Apparently, a certain amount of adsorbates was still present on graphene surface after the vacuum condition had been achieved: during the temperature-dependent measurements, ratio of $2 \mathrm{D}$ and $\mathrm{G}$ peak intensities $\mathrm{I}_{2 \mathrm{D}} / \mathrm{I}_{\mathrm{G}}$ were smaller than $\sim 3$ typical for completely undoped graphene [23]; however, near-constant overall $\mathrm{I}_{2 \mathrm{D}} / \mathrm{I}_{\mathrm{G}}$ values for all samples during the experiment confirmed the atmospheric adsorption to introduce constant contribution to the obtained dependencies, its value obtainable from $\mathrm{I}_{2 \mathrm{D}} / \mathrm{I}_{\mathrm{G}}$ and $\mathrm{G}(2 \mathrm{D})$ position dependencies on charge carrier density [23].

The measured $G$ and $2 D$ peak position temperature shifts were fitted with the curves obtained through the above calculations, anharmonic constants being the fitting parameters; the model calculation results are presented in Fig. 2 as solid lines. As seen, the utilized method describes dependencies for undoped graphene on dielectric substrates well. In case of $G$ and $2 D$ peak shifts for graphene on copper and 2D peak shift for doped graphene, there is a noticeable discrepancy between the measurement results and the calculated curve at temperatures below $\sim 100 \mathrm{~K}$. This can be attributed to changes in the EPC of doped graphene or graphene supported by metals: the effects of a metallic substrate, as well as additional charge carriers introduced through graphene doping, knowingly lead to EPC screening in graphene [39,40]. Taking into account that during the calculation, we used EPC contribution temperature dependencies theoretically obtained without taking substrate or doping into account [32], this results in a divergence of calculated line with the experimental points at low temperatures.

The obtained constants representing the contributions of 3- and 4-phonon processes to the observed frequency shift for the experimental graphene are given in Table 1 . The extracted values of these parameters straightforwardly represent contributions of each process to the lineshift: since the exponential parts of the expression (3) are multiplied by the constants, larger absolute values of $A_{3-p h}^{G, 2 D}$ and $B_{4-p h}^{G, 2 D}$ correspond to larger contributions of three- and four-phonon processes, respectively.

Considering peak position temperature dependencies given in Fig. 2 and parameters presented in Table 1, an exceedingly explicit effect of the substrate on graphene phonon properties is observed. The contributions of 3-phonon processes to the anharmonic mode frequency shift are positive and smaller comparing to the negative values of $B_{4-p h}^{G}$ and $B_{4-p h}^{2 D}$, which is fully consistent with theory [30,32] - the 3-phonon interactions lead to a slight increase of the peak position as the temperature rises, while the 4-phonon processes dominate with a stronger decrease, consequently observed on the experimental curves. Values of $A_{3-p h}^{G}$ and $B_{4-p h}^{G}$ obtained for graphene on $\mathrm{SiO}_{2} / \mathrm{Si}$ and $\mathrm{Al}_{2} \mathrm{O}_{3}$ are quantitatively close to those measured and theoretically calculated for unsupported graphene $[25,32]$, while for graphene as-grown on copper, $B_{4-p h}^{2 D}$ is about 40 times larger than that experimentally obtained for unsupported graphene from Ref. [25] and up to 70 times larger than the theoretical value, calculated without taking substrate into account [32]. The analysis of such an extremely strong substrate effect on the anharmonic constants requires paying close attention to the interface-related phonon effects in graphene.

Graphene-substrate interaction is known to effectively manifest itself in (i) phonon mode energy exchange due to leaking of phonons through the interface [15] or vibration scattering on the interface [16] and (ii) oscillating atom mass center displacement due to charge transfer effects $[13,41]$. These effects naturally lead to the deviations of vibrational force constants and atomic displacements from equilibrium positions typical for free-standing graphene, correspondingly affecting vibrational Hamiltonian and leading to the anharmonicity increase, which emphasizes the importance of choosing the suitable substrate for a specific graphene application.

Comparing the obtained results for as-grown and transferred graphene on copper, one can notice a significant difference in the values of anharmonic constants, which are strengthened for the former by $13-590 \mathrm{~cm}^{-1}$. This fact is apparently connected with a possibility of additional chemical bonding between the as-grown graphene and copper [36] which can lead to a partial inapplicability of the Van-der-Waals graphene-substrate interaction model for this case. Although both theoretical calculations [34] for graphene on copper and experimental measurements for as-grown graphene [37] give similar results of adhesion energy, chemical bonds still can lead to a locally increased strain, as well as to the deviations from the equilibrium graphene-substrate distance, affecting the intensity of phonon scattering $[15,16]$ and out-ofplane phonon exchange [15] at the interface, which results in larger values of anharmonic constants and, consequently, stronger influence of both terms of equation (3). It should be noted that though $G$ and 2D peak Raman processes involve the in-plane longitudinal (iLO) and transverse (iTO) optical modes, these modes still can participate in the anharmonic interactions with the out-ofplane phonons [11].

Residual chemical bonds are terminated during the transfer process, leading to a pure copper effect for graphene transferred to $\mathrm{Cu}$ substrate, which is clearly manifested when comparing the obtained anharmonic constants in Table 1 with those for graphene on $\mathrm{SiO}_{2} / \mathrm{Si}$ - for the latter, the values are 1.1-2.6 times smaller. As already mentioned, graphene-copper interaction has the energy of $0.72 \mathrm{~J} / \mathrm{m}^{2}$, while that for $\mathrm{SiO}_{2} / \mathrm{Si}$ is $0.45 \mathrm{~J} / \mathrm{m}^{2}$ [34]. It is known that for 
Table 1

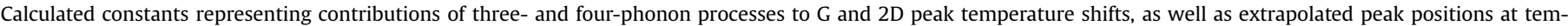
peratures close to absolute zero.

\begin{tabular}{|c|c|c|c|c|c|c|}
\hline Substrate & $\omega_{0}^{G}, \mathrm{~cm}^{-1}$ & $A_{3-p h}^{G}, \mathrm{~cm}^{-1}$ & $B_{4-p h}^{G}, \mathrm{~cm}^{-1}$ & $\omega_{0}^{2 D}, \mathrm{~cm}^{-1}$ & $A_{3-p h}^{2 D}, \mathrm{~cm}^{-1}$ & $B_{4-p h}^{2 D}, \mathrm{~cm}^{-1}$ \\
\hline Copper (as-grown) & 1624 & 39 & -84 & 2771 & 736 & -849 \\
\hline Copper (transferred) & 1592 & 26 & -35 & 2719 & 209 & -260 \\
\hline $\mathrm{SiO}_{2} / \mathrm{Si}$ & 1592 & 10 & -21 & 2715 & 191 & -231 \\
\hline $\mathrm{Al}_{2} \mathrm{O}_{3}$ & 1585 & 8 & -12 & 2709 & 162 & -214 \\
\hline $\mathrm{SiO}_{2} / \mathrm{Si}$ (doped) & 1597 & 10 & -27 & 2715 & 108 & -150 \\
\hline
\end{tabular}

stronger graphene-substrate interactions, optical modes generally tend to soften [13]: as graphene gets adsorbed on copper, out-ofplane optical (oTO) and iLO phonon mode energies at the Brillouin Zone center are reduced by $\sim 3$ and $\sim 8 \mathrm{meV}$, while at $\mathrm{K}$ point the shifts are $\sim 4$ and $\sim 1 \mathrm{meV}$, respectively [42,43]. This suggests that not only the out-of-plane modes can experience scattering at the interface, but the in-plane modes as well, as it was shown in Ref. [16], although the effect can be expected more pronounced for the former since they correspond to vertical atom vibrations [15]. In addition, one should not exclude the influence of similar acoustic phonon mode effects through the coupling between optical and acoustic phonons. A stronger graphene-metal interaction obviously implies more pronounced manifestation of the described effects when compared to $\mathrm{SiO}_{2} / \mathrm{Si}$.

Phonons can interact with other quasi-particles such as charge carriers, leading to a reduction of graphene electrical conductivity through scattering processes. Despite EPC in graphene on metals can be reduced or even annihilated through substrate electron coupling with graphene adlayer [44], charge transfer from copper to graphene expectedly leads to a selective $\pi$ bond softening in graphene $[13,41]$ - consequently, this changes force constants related to atomic motion, affecting the vibrational Hamiltonian.

The obtained anharmonic constants for graphene on $\mathrm{SiO}_{2} / \mathrm{Si}$ and $\mathrm{Al}_{2} \mathrm{O}_{3}$ have considerably close values. For the latter, the influence of both 3- and 4-phonon processes at Brillouin zone center (G peak) is the weakest among the examined samples, with all values of anharmonic constants being slightly smaller than those obtained for undoped graphene on $\mathrm{SiO}_{2} / \mathrm{Si}$. Due to a stronger lattice mismatch for graphene on $\mathrm{Al}_{2} \mathrm{O}_{3}$ than on $\mathrm{SiO}_{2} / \mathrm{Si}[45,46]$, the effect of phonon exchange through the interface can be expected less pronounced for the former, while the mechanical strain influence varies weakly with the temperature, according to the results of our strain-related peak shift calculations. At the same time, taking into account close values of graphene-substrate interaction energy $0.45 \mathrm{~J} / \mathrm{m}^{2}$ for $\mathrm{SiO}_{2} / \mathrm{Si} \mathrm{[34]} \mathrm{and} 0.47 \mathrm{~J} / \mathrm{m}^{2}$ for $\mathrm{Al}_{2} \mathrm{O}_{3}$ [35] - the close values of the anharmonic constants obtained for these samples confirm logical-to-expect relationship between the interaction strength and the extent of the substrate effect.

Doping of graphene tends to introduce additional distortions into the lattice of the material. According to the obtained values of anharmonic constants for doped graphene on $\mathrm{SiO}_{2} / \mathrm{Si}$, the effect is expressed for $B_{4-p h}^{G}$ comparing to that for undoped graphene on the same substrate (Table 1 ). At the same time, both constants for the double-resonance process at $\mathrm{K}$ point of the Brillouin Zone (2D peak) turned out to be softened, possibly due to electronic dispersion distortion in the vicinity of Dirac point as a result of doping: except contributing to phonon scattering, nitrogen atoms introduced in graphene act as a source of both localized and delocalized electrons [47]. For undoped graphene, a high DOS below the conduction band edge in silicon dioxide is known to donate electrons, balancing chemical potential at the interface [48], while for nitrogen-doped graphene, this effect can be softened due to an already increased electron concentration, leading to a reduction of scattering processes in the vicinity of $\mathrm{K}$ point which are strongly sensitive to charge carrier effects [23].

The results of a similar experiment for sample heating (i.e. carried out in the opposite direction), as well as the corresponding discussion, are provided in Appendix B. The anharmonic constants calculated for this case show similar trends, demonstrating that in general the observed features tend to preserve independently of condition change direction.

Fig. 3 shows temperature variations of $G$ and 2D peak linewidths with the temperature. As seen, the dependencies tend to be substrate-dependent again, with the broadening for as-grown graphene on copper being the most affected. $G$ peak linewidth variations for graphene on $\mathrm{Al}_{2} \mathrm{O}_{3}$, as well as for doped graphene, are within the error in this temperature range. The same is valid for 2D peak linewidth variations for both doped and undoped graphene transferred to $\mathrm{SiO}_{2} / \mathrm{Si}$.

According to the calculations for unsupported graphene presented in Ref. [32], EPC contribution to the linewidth of $G$ and 2D peaks can be considered constant in the low-temperature range (below $\sim 300 \mathrm{~K}$ ), with the values of $\Gamma_{e l-p h}^{G}(0) \approx 9.1 \mathrm{~cm}^{-1}$ and $\Gamma_{e l-p h}^{2 D}(0) \approx 22.0 \mathrm{~cm}^{-1}$. The same is valid for $G$ peak linewidth variations due to anharmonic phonon-phonon interactions $\left(T_{p h-p h}^{G}(0) \approx 7.2 \mathrm{~cm}^{-1}\right)$. However, a linear change in 2D peak linewidth driven by the latter is expected $[25,32]$. Thus, according to theory for unsupported graphene [32], temperature behavior of $G$ and 2D peak linewidths at temperatures below $300 \mathrm{~K}$ can be described by the following expressions:

$$
\begin{aligned}
& \Gamma^{G}(T)=\Gamma_{p h-p h}^{G}(0)+\Gamma_{e l-p h}^{G}(0) \approx \text { const }=16.3 \mathrm{~cm}^{-1} \\
& \Gamma^{2 D}(T)=C_{p h-p h}^{2 D} T+\Gamma_{p h-p h}^{2 D}(0)+\Gamma_{e l-p h}^{2 D}(0)
\end{aligned}
$$

where $\Gamma_{p h-p h}^{2 D}(0) \approx 2.5 \mathrm{~cm}^{-1}$ [32] is the initial anharmonic phonon process contribution to $2 \mathrm{D}$ peak FWHM at temperature close to absolute zero; $C_{p h-p h}^{2 D}$ is a linear coefficient of linewidth temperature shift due to anharmonic phonon interactions (the 3- and 4phonon contributions are not distinguishable through the approximation in this temperature range due to the linear dependence).

As seen from expression (7), a superposition of several processes leads to the expected linear change of $2 \mathrm{D}$ peak linewidth with the temperature, which is confirmed by the obtained dependencies in Fig. 3 for all undoped samples. At the same time, theory for unsupported graphene [32] does not predict any changes in G peak linewidth, while the opposite was observed during the experiment (Fig. 3) for undoped graphene on copper and $\mathrm{SiO}_{2} / \mathrm{Si}$. Although there is no theoretical background for using the linear approximation of $G$ peak linewidth temperature dependence in our case (no broadening was expected in this temperature range and the expressions applicable for higher temperatures [30,32] naturally 

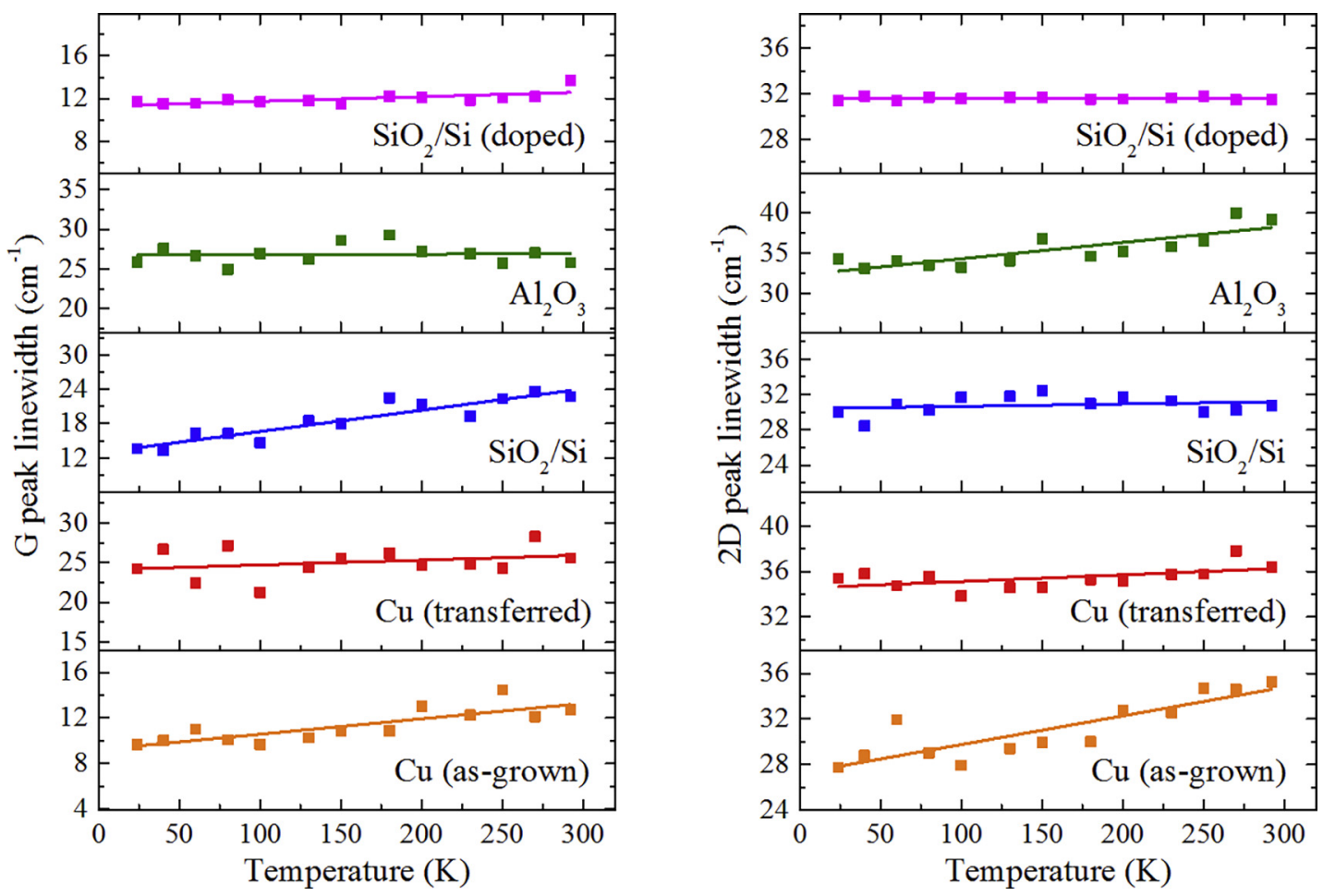

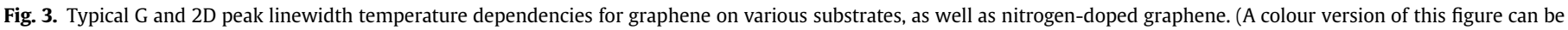
viewed online.)

did not match the points), a linear fit was utilized for the uniformity of the presented data, as well as simplicity of comparison with 2D peak behavior.

For as-grown graphene on copper, both $G$ and 2D peak linewidths varied with the temperature with the coefficients of $1.4 \cdot 10^{-2}$ and $2.5 \cdot 10^{-2} \mathrm{~cm}^{-1} \mathrm{~K}^{-1}$, respectively. Transfer to copper demonstrated softening of these parameters down to the same value of $0.6 \cdot 10^{-2} \mathrm{~cm}^{-1} \mathrm{~K}^{-1}$. The difference can be primarily attributed to already discussed deviations of graphene-substrate distance for as-grown graphene, leading to stronger phonon interactions at the interface in this case. Besides, the presence of $G$ peak linewidth variation implies a possible impact of charge transfer effects: while the EPC contribution is expected to be constant in case of unsupported graphene [32], it can be reduced through substrate electron coupling with graphene adlayer as mentioned previously [44]. At the same time, graphene-substrate distance fluctuations with the temperature [49] can lead to a temperature-dependent change in this effect (and consequently, in the second term in (6)) more pronounced for as-grown graphene which is natural considering the above discussion.

Graphene on $\mathrm{SiO}_{2} / \mathrm{Si}$ demonstrated strong $\mathrm{G}$ peak linewidth decrease as the temperature decreased with the coefficient of $3.7 \cdot 10^{-2} \mathrm{~cm}^{-1} \mathrm{~K}^{-1}$, while that for $2 \mathrm{D}$ peak had a value within the error. In contrast to the position temperature shift (Fig. 2), a pronounced $G$ peak linewidth temperature dependence in this case looks quite unexpected considering theoretical predictions [32], and is possibly connected to electron transfer from the substrate [48] that strongly affects EPC contribution in (6) and correspondingly, $\mathrm{G}$ peak linewidth [50], while 2D peak anharmonic broadening is clearly suppressed. For graphene on $\mathrm{Al}_{2} \mathrm{O}_{3}$, an inverse situation is observed - greater coefficient of $2.0 \cdot 10^{-2} \mathrm{~cm}^{-1} \mathrm{~K}^{-1}$ for 2D peak linewidth and negligible within the error $G$ peak linewidth variation - that is in a strong agreement with both theoretical [32] and experimental [25] results presented in the literature, additionally showing that this substrate introduces the smallest effect on graphene properties among the analyzed samples.

For doped graphene, negligible within the experimental error variations of both $G$ and 2D peak linewidths are other arguments in favor of strong electron-phonon coupling effect on the linewidth dependencies. Introducing additional charge carriers, in this case, leads to softening of EPC, which results in the absence of the experimental broadening. This is a favorable observation, taking into account the strong and often undesired influence of both EPC and phonon anharmonicities on carrier transport in graphene devices $[6,30]$.

In general, interaction with a substrate brings significant changes to graphene phonon properties, sometimes comparable to the interlayer interaction in bilayer or few-layer graphene [51] in terms of phonon-phonon and electron-phonon processes. As graphene gets adsorbed on the substrate, phonon relaxation times get reduced [16,52]; phonon leaking through the interface creates additional charge carrier scattering source, considering which is especially useful when a sensitive calculation is needed for a nanoelectronic device engineering. Among the substrates analyzed, the "least anharmonic" behavior was observed for graphene on $\mathrm{Al}_{2} \mathrm{O}_{3}$, while for a relatively strong graphene-copper interaction, the effect was significant. Besides, different behavior was observed for doped and undoped graphene on the same substrate. Supporting graphene by substrates with larger values of the interaction energy opens up new prospects for modification of phonon anharmonicities in graphene, important for both further studies of graphene high-order phonon properties and controllable tuning of scattering processes in the material. 


\section{Conclusion}

Temperature-dependent Raman measurements were performed for graphene as-grown on copper, transferred to $\mathrm{SiO}_{2} / \mathrm{Si}$, copper and $\mathrm{Al}_{2} \mathrm{O}_{3}$, and nitrogen-doped graphene on $\mathrm{SiO}_{2} / \mathrm{Si}$ in the temperature range of $20-294 \mathrm{~K}$. Different $\mathrm{G}$ and $2 \mathrm{D}$ peak position and linewidth temperature dependencies were obtained and analyzed taking into account contributions of anharmonic phonon effects, graphene thermal expansion, substrate-induced strain and electron-phonon coupling. Determined anharmonic constants for 3- and 4-phonon processes in graphene were found to be strongly influenced by substrate and doping. The values obtained from G peak shift for undoped graphene on dielectric substrates were quantitatively close to the experimental results for unsupported graphene and theoretical predictions reported in the literature, while the constants for graphene as-grown on copper were almost two orders of magnitude greater. The results were analyzed in terms of substrate and doping influence on phonon and electronic properties of graphene through phonon scattering and phonon exchange at the interface, graphene-substrate charge transfer and EPC screening effects. Present study is useful for taking into account anharmonic phonon effects on phonon and electronic transport in graphene during future graphene-based nanoelectronic device engineering.

\section{Acknowledgments}

This work was supported by Belarusian State Program for Research "Photonics, opto- and microelectronics" and Russian Foundation of Basic Researches (individual project 16-33-60229), as well as partially supported by Basic Science Research Program through the National Research Foundation of Korea funded by the Ministry of Education (No. 2017R1D1A1B03035102 and 2017R1D1A1B03032759). The authors of this work are grateful to Prof. L.V. Yashina and Ph.D. Student A. I. Belova for XPS studies of nitrogen-doped graphene.

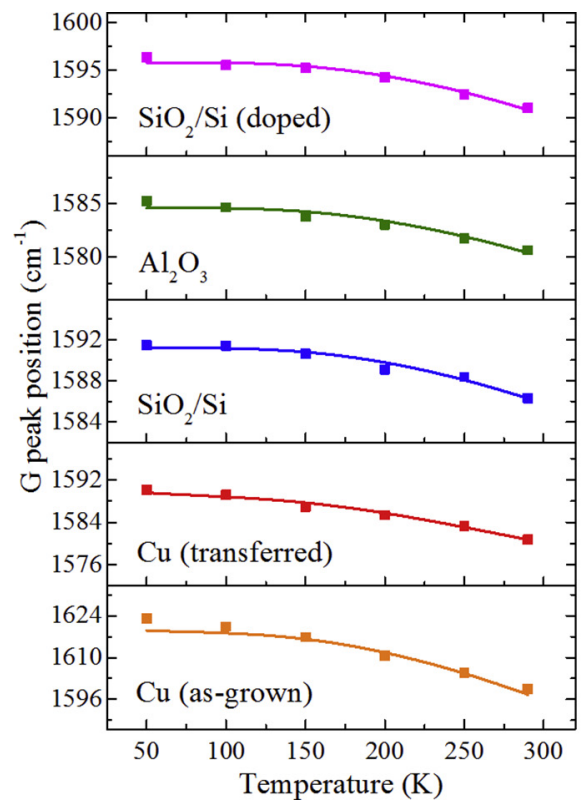

\section{Appendix A}

Other works on the subject usually present Raman temperature shifts with the linear approximations [27,53-56]. Although this is not the most accurate way to describe this effect, we performed linear approximations in order to make our results more comparable with those obtained by other authors. The obtained peak shift coefficients $\chi^{G, 2 D}$, as well as extrapolated peak positions at temperatures close to absolute zero $\omega_{0}^{G, 2 D}$, are given in Table A1.

Table A1

Coefficients of G and 2D peak temperature shifts, as well as extrapolated peak positions at temperatures close to absolute zero, obtained from linear approximations for graphene on various substrates and nitrogen-doped graphene during the cooling experiments.

\begin{tabular}{lllll}
\hline Substrate & $\omega_{0}^{G}, \mathrm{~cm}^{-1}$ & $\chi^{G}, \mathrm{~cm}^{-1} \cdot \mathrm{K}^{-1}$ & $\omega_{0}^{2 D}, \mathrm{~cm}^{-1}$ & $\chi^{2 D}, \mathrm{~cm}^{-1} \cdot \mathrm{K}^{-1}$ \\
\hline Copper (as-grown) & 1627 & -0.076 & 2778 & -0.179 \\
Copper (transferred) & 1593 & -0.028 & 2721 & -0.069 \\
$\mathrm{SiO}_{2} / \mathrm{Si}$ & 1593 & -0.018 & 2716 & -0.028 \\
$\mathrm{Al}_{2} \mathrm{O}_{3}$ & 1586 & -0.011 & 2709 & -0.028 \\
$\mathrm{SiO}_{2} / \mathrm{Si}$ (doped) & 1598 & -0.023 & 2717 & -0.036 \\
\hline
\end{tabular}

As seen, linear approximations still show an explicit substrate influence on this effect. The obtained values are in agreement with other experimental data presented in the literature [27,53,54]. The small differences can be mainly attributed to various temperature ranges of investigation in different works: at higher temperatures, Raman peak shift is more linearized, and thus fits to a conventional straight-line approximation better.

\section{Appendix B}

Immediately after low-temperature measurements with graphene cooling, the reverse experiment with heating was conducted for each sample. The obtained $G$ and $2 \mathrm{D}$ peak shift temperature dependencies are presented in Fig. B1.

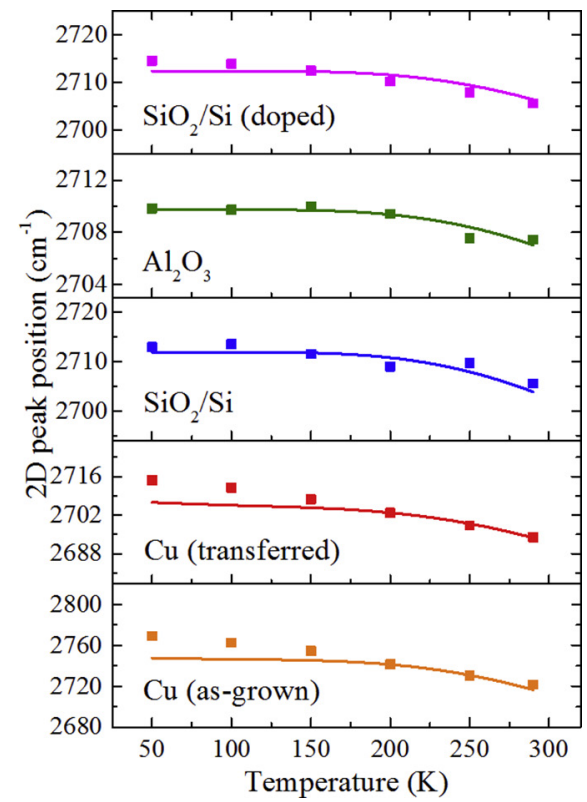

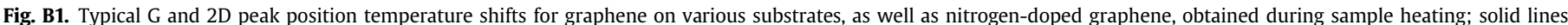
represent the results of a model calculation. 
On the basis of the dependencies obtained, the anharmonic constants were calculated in a similar way to those determined from the cooling experiments. The obtained parameters are presented in Table B1. Again, the model curves show the already discussed discrepancy at temperatures below $100 \mathrm{~K}$ of G and 2D peak shifts for graphene as-grown copper and 2D peak shifts for graphene doped or transferred on copper, though the curve fits $G$ peak shift of the latter well in this case.

\section{Table B1}

Constants representing contributions of three- and four-phonon processes to $\mathrm{G}$ and 2D peak temperature shifts, as well as extrapolated peak positions at temperatures close to absolute zero, calculated during the heating experiment.

\begin{tabular}{lllll}
\hline Substrate & $A_{3-p h}^{G}, \mathrm{~cm}^{-1}$ & $B_{4-p h}^{G}, \mathrm{~cm}^{-1}$ & $A_{3-p h}^{2 D}, \mathrm{~cm}^{-1}$ & $B_{4-p h}^{2 D}, \mathrm{~cm}^{-1}$ \\
\hline Copper (as-grown) & 37 & -95 & 653 & -809 \\
Copper (transferred) & 25 & -44 & 236 & -290 \\
$\mathrm{SiO}_{2} / \mathrm{Si}$ & 11 & -21 & 190 & -230 \\
$\mathrm{Al}_{2} \mathrm{O}_{3}$ & 14 & -19 & 42 & -76 \\
$\mathrm{SiO} 2 / \mathrm{Si}$ (doped) & 5 & -20 & 118 & -170 \\
\hline
\end{tabular}

All trends for anharmonic constants presented in Table 1 (cooling) and $\mathrm{B} 1$ (heating) demonstrate considerable similarity, except for $A_{3-p h}^{2 D}$ and $B_{4-p h}^{2 D}$ graphene on $\mathrm{Al}_{2} \mathrm{O}_{3}$. The general similarity of the constants obtained under different conditions (i.e., heating) indicates the stability of the phonon features formed as a result of graphene-substrate interaction. Regardless of the direction of phonon system evolution, substrate-related anharmonic effects repeatably affect its dynamics in a similar way. At the same time, the softening of parameters obtained from 2D peak shift for graphene on $\mathrm{Al}_{2} \mathrm{O}_{3}$ by $122-138 \mathrm{~cm}^{-1}$ can be attributed to graphenesubstrate interaction relaxation during the experiment. Taking into account both weak interaction energy [35] and strong lattice mismatch [45] in this case, graphene subjection to sequential environmental cooling and laser-induced local heating can lead to a regionally weakened interaction, correspondingly softening the substrate effect on the mode anharmonicity. This results in a deviation of anharmonic constants for cooling and heating experiments for a more sensitive to graphene-substrate interaction iTO mode at the Brillouin Zone edge (2D peak).

\section{References}

[1] A.K. Geim, K.S. Novoselov, Nat. Mater. 6 (2007) 183-191.

[2] A.A. Balandin, S. Ghosh, W. Bao, I. Calizo, D. Teweldebrhan, F. Miao, C.N. Lau, Nano Lett. 8 (2008) 902-907.

[3] A.A. Balandin, Nat. Mater. 10 (2011) 569-581.

[4] D.L. Nika, A.A. Balandin, Rep. Prog. Phys. 80 (036502) (2017).

[5] T.Y. Kim, C.-H. Park, N. Marzari, Nano Lett. 16 (2016) 2439.

[6] T. Gunst, T. Markussen, K. Stokbro, M. Brandbyge, Phys. Rev. B 93 (2016) 035414.

[7] C.-H. Park, N. Bonini, T. Sohier, G. Samsonidze, B. Kozinsky, M. Calandra, F. Mauri, N. Marzari, Nano Lett. 14 (2014) 1113.

[8] D.L. Nika, E.P. Pokatilov, A.S. Askerov, A.A. Balandin, Phys. Rev. B 79 (2009) 155413.

[9] L. Lindsay, D.A. Broido, N. Mingo, Phys. Rev. B 82 (2010) 115427.

[10] L. Lindsay, W. Li, J. Carrete, N. Mingo, D.A. Broido, T.L. Reinecke, Phys. Rev. B 89 (2014) 155426.
[11] D.L. Nika, A.A. Balandin, J. Phys. Condens. Matter 24 (2012) 233203.

[12] H. Malekpour, A.A. Balandin, J. Raman Spectrosc. 49 (2018) 106-120.

[13] A.A. Taleb, D. Farías, J. Phys. Condens. Matter 28 (2016) 103005.

[14] Z.-Y. Ong, E. Pop, Phys. Rev. B 84 (2011) 075471.

[15] J.H. Seol, I. Joa, A.L. Moore, L. Lindsay, D. Broido, N. Mingo, R.S. Ruoff, L. Shi, Science 328 (2010) 213.

[16] B. Qiu, X. Ruan, Appl. Phys. Lett. 100 (2012) 193101.

[17] A.C. Ferrari, D.M. Basko, Nat. Nanotechnol. 8 (2013) 235.

[18] F. Güneș, G.H. Han, K.K. Kim, E.S. Kim, S.J. Chae, M.H. Park, H.-K. Jeong, S.C. Lim, Y.H. Lee, Nano 4 (2009) 83.

[19] X. Liang, B.A. Sperling, I. Calizo, G. Cheng, C.A. Hacker, Q. Zhang, Y. Obeng, K. Yan, H. Peng, Q. Li, X. Zhu, H. Yuan, A.R. Walker, Z. Liu, L.M. Peng, C.A. Richter, ACS Nano 5 (2011) 9144.

[20] L. Gao, G.-X. Ni, Y. Liu, B. Liu, A.H. Castro Neto, K.P. Loh, Nature 505 (2014) 190.

[21] M. Her, R. Beams, L. Novotny, Phys. Lett. 377 (2013) 1455.

[22] H. Wang, T. Maiyalagan, X. Wang, ACS Catal. 2 (2012) 781.

[23] R. Beams, L.G. Cançado, L. Novotny, J. Phys. Condens. Matter 27 (2015) 083002.

[24] T.M.G. Mohiuddin, A. Lombardo, R.R. Nair, A. Bonetti, G. Savini, R. Jalil, N. Bonini, D.M. Basko, C. Galiotis, N. Marzari, K.S. Novoselov, A.K. Geim, A.C. Ferrari, Phys. Rev. B 79 (2009) 205433.

[25] J. Lin, L. Guo, Q. Huang, Y. Jia, K. Li, X. Lai, X. Chen, Phys. Rev. B 83 (2011) 125430.

[26] S. Mann, V.K. Jindal, Mater. Res. Express 4 (2017) 075038.

[27] S. Tian, Y. Yang, Z. Liu, C. Wang, R. Pan, C. Gu, J. Li, Carbon 104 (2016) 27.

[28] E.A. Kolesov, M.S. Tivanov, O.V. Korolik, O.O. Kapitanova, X. Fu, H.D. Cho, T.W. Kang, G.N. Panin, Beilstein J. Nanotechnol. 9 (2018) 704-710.

[29] M. Lazzeri, M. Calandra, F. Mauri, Phys. Rev. B 68 (2003), 220509(R).

[30] N. Bonini, M. Lazzeri, N. Marzari, F. Mauri, Phys. Rev. Lett. 99 (2007) 176802.

[31] C. Postmus, J.R. Ferraro, Phys. Rev. 174 (1968) 983.

[32] A.T. Apostolov, I.N. Apostolova, J.M. Wesselinowa, J. Phys. Condens. Matter 24 (2012) 235401

[33] J.-W. Jiang, J.-S. Wang, B. Li, Phys. Rev. B 80 (2009) 205429.

[34] Y. He, W.F. Chen, W.B. Yu, G. Ouyang, G.W. Yang, Sci. Rep. 3 (2013) 2660.

[35] B. Huang, Q. Xu, S.-H. Wei, Phys. Rev. B 84 (2011) 155406.

[36] S. Das, D. Lahiri, D.Y. Lee, A. Agarwal, W. Choi, Carbon 59 (2013) 121.

[37] T. Yoon, W.C. Shin, T.Y. Kim, J.H. Mun, T.-S. Kim, B.J. Cho, Nano Lett. 12 (2012) 1448.

[38] D. Yoon, Y. Son, H. Cheong, Nano Lett. 11 (2011) 3227.

[39] D.A. Siegel, C. Hwang, A.V. Fedorov, A. Lanzara, N. J. Phys. 14 (2012) 095006.

[40] C. Attaccalite, L. Wirtz, M. Lazzeri, F. Mauri, A. Rubio, Nano Lett. 10 (2010) 1172.

[41] P.A. Khomyakov, G. Giovannetti, P.C. Rusu, G. Brocks, J. van den Brink, P.J. Kelly, Phys. Rev. B 79 (2009) 195425.

[42] J.A. Yan, W.Y. Ruan, M.Y. Chou, Phys. Rev. B 77 (2008) 125401.

[43] L. Chen, Z. Huang, S. Kumar, Appl. Phys. Lett. 103 (2013) 123110.

[44] A. Allard, L. Wirtz, Nano Lett. 10 (2010) 4335.

[45] X. Chen, B. Wu, Y. Liu, Chem. Soc. Rev. 45 (2016) 2057.

[46] W. Gao, P. Xiao, G. Henkelman, K.M. Liechti, R. Huang, J. Phys. D Appl. Phys. 47 (2014) 255301

[47] F. Joucken, Y. Tison, P. Le Fèvre, A. Tejeda, A. Taleb-Ibrahimi, E. Conrad, V. Repain, C. Chacon, A. Bellec, Y. Girard, S. Rousset, J. Ghijsen, R. Sporken, H. Amara, F. Ducastelle, J. Lagoute, Sci. Rep. 5 (2015) 14564.

[48] H.E. Romero, N. Shen, P. Joshi, H.R. Gutierrez, S.A. Tadigadapa, J.O. Sofo, P.C. Eklund, ACS Nano 2 (2008) 2037.

[49] S. Ulstrup, M. Bianchi, R. Hatch, D. Guan, A. Baraldi, D. Alfè, L. Hornekær, P. Hofmann, Phys. Rev. B 86 (2012), 161402(R).

[50] J. Koivistoinen, P. Myllyperkiö, M. Pettersson, J. Phys. Chem. Lett. 8 (2017) 4108.

[51] K. Kaviyarasu, E. Manikandan, J. Kennedy, M. Maaza, Journ. Mat. Sci. Mater. Electr 27 (2016) 13080.

[52] F. de Juan, A. Politano, G. Chiarello, H.A. Fertig, Carbon 85 (2015) 225.

[53] I. Calizo, A.A. Balandin, W. Bao, F. Miao, C.N. Lau, Nano Lett. 7 (2007) 2645.

[54] W. Wang, Q. Peng, Y. Dai, Z. Qian, S. Liu, J. Mater. Sci. Mater. Electron. 27 (2016) 3888.

[55] E.A. Kolesov, M.S. Tivanov, O.V. Korolik, A.M. Saad, I.V. Komissarov, Carbon 111 (2017) 587.

[56] J. Judek, A.P. Gertych, M. Krajewski, K. Czerniak, A. Łapińska, J. Sobieski, M. Zdrojek, Carbon 124 (2017) 1. 